gende Verhältnis zwischen Gesundheit und Leistungsfähigkeit, die die Subjekte in ihren Berichten nicht thematisiert haben, sowie im Hinblick auf die Praxis des Sich-Selbst-Gefährdens, das in dem Interdiskurs stark, doch in den Selbsterfahrungsberichten nicht zentral und explizit thematisiert wurde. Schließlich wurde der Präventionsdispositiv in größere soziale Wandlungen und gesamtgesellschaftliche Kontexte gesetzt. Hier wurde die Wandlung der Subjektform beobachtet, durch die die Subjekte zur Selbstgefährdung einerseits und Selbstprävention andererseits angeleitet werden. Diese paradoxe Konstellation verbindet sich mit einer sicherheits- und präventionsgeleiteten Regierungskultur, die einen Zustand herstellt, in dem sich die Subjekte nach dem Schutz vor sich selbst sehnen und diesen von außen erwarten. Alle Elemente des Präventionsdispositivs verbinden sich in dem Maße, wie das diskursive Wissen um Prävention in ihnen enthalten ist und zwischen ihnen zirkuliert. Außerdem bedingen sie sich gegenseitig und setzen sich voraus, indem, z.B. für die Benutzung eines Geräts (Fitness-Tracker) eine entsprechende diskursive Software (App), ein bestimmter Subjektmodus (optimierendes Subjekt) und eine einverleibte nicht-diskursive Praxis (Sport treiben) vonnöten ist.

Die untersuchte Landschaft des Präventionsdispositivs wurde im konkreten Zeitraum (Ende des 20. und Anfang des 21. Jahrhunderts) und im bestimmten gesellschaftstheoretischen Kontext (Neoliberalismus) beschrieben. Sie kann unterschiedliche Formen annehmen, je nachdem, ob sie Praktiken, Diskurse, Subjektformen oder Gegenstände erzeugt. Die im Rahmen dieser Untersuchung beschriebenen Elemente entsprechen keineswegs einer erschöpfenden Darstellung des Präventionsdispositivs, sondern stellen vielmehr einen Faden dar, entlang welchem sein Wirken beobachtet und beschrieben werden kann. Das heißt zugleich, dass sich das Präventionsdispositiv in Zeit und Raum weiterentfalten und modifizieren kann, so wie dies am Beispiel der Disziplin und des Sicherheitsdispositivs, die eine Art Vorläufer des Präventionsdispositivs darstellen, sichtbar wurde. Die Untersuchung seiner Landschaft hat das Ziel erreicht, die Etablierung der BurnoutPrävention als einer immanenten Regierungsweise zu beschreiben und ihre gesellschaftliche Verankerung und Verselbstständigung nachzuweisen.

\title{
2 Machteffekte und Widerstandspotentiale
}

Als zweites wurde mit der kritischen Analyse der Burnout-Prävention das Ziel verfolgt, die im Präventionsdispositiv wirkenden Machteffekte, sowie die unterschiedlichen Arten von Widerstandspotentialen und -optionen, die das Präventionsdispositiv vorstrukturiert, zulässt oder zu verhindern sucht, zu erhellen.

Wie bei der Beschreibung der Dispositivanalyse nach Michel Foucault gezeigt wurde, reagiert das Dispositiv auf einen Notstand, d.h. auf eine Situation, die das herrschende Regierungsregime bedroht. Im Rahmen der vorliegenden Untersu- 
chung wurde der Neoliberalismus als das dominierende politische Projekt bzw. das herrschende Regierungsregime bezeichnet. Demnach stellte sich die Frage nach dem Zusammenhang zwischen der Burnout-Prävention, als Variation eines Präventionsdispositivs, und dem Neoliberalismus, als dem dominanten Regierungsregime. Während der Analysen wurde deutlich, dass die Prävention des BurnoutSyndroms eng mit den Anforderungen an Leistungs- und Wettbewerbsfähigkeit verknüpft wird. So haben beispielsweise HRV-Messgeräte den Anspruch erhoben, auch die Schlafqualität zu messen und angesichts einer ganztägigen, ausbalancierten Verteilung von Leistung und Ruhepausen, die eigenen Optimierungsmöglichkeiten aufzuzeigen. Auch die Einrichtung des Büroraumes, welche zur entspannten Wachheit und damit zur besseren Arbeitsleistung beitragen soll - »Ich muss mich entspannen, um meine Leistungsfähigkeit weiter zu steigern« (Hillert/Koch/Lehr 2018: 43 [Herv. i. O.]) -, zeugt von dem Versuch, die Prävention als Sicherung der neoliberalen Arbeits- und Regierungsweise zu gebrauchen. Schließlich unterstützt den Wettbewerb auch die Praxis der Gamification, die die Ziele des Unternehmens mit den eigenen Leistungs- und Erfolgszielen verschmelzen und dadurch die neoliberale Optimierung ungestört wirken lässt.

Das Präventionsdispositiv dient daher als ein Schutzmechanismus, der das neoliberale Regierungsregime vor seiner Hinterfragung oder gar seiner Auflösung behüten soll. So gesehen verhindert dieses Dispositiv weniger die tatsächlichen Ursachen - nicht $\mathrm{zu}$ leistende Leistungen, Individualisierung gesellschaftlicher Probleme, Verbreitung der Marktlogik in öffentliche und private Lebensbereiche usw. -, die zum Ausbrennen vieler Individuen führen, als vielmehr die Möglichkeit, diese Ursachen, und damit das herrschende Regime, radikal hinterfragen zu können.

Dieser Machtmechanismus wird dadurch gestärkt, dass er die disponierenden und disponierten Subjektivitäten nicht klar voneinander trennt, sondern sie in eine und dieselbe Subjektform verlagert. So besteht die Aufgabe des im Präventionsdispositiv erzeugten Subjekts darin, sich selbst gleichzeitig als Problem und als Lösung, als Gefährdung und als Präventionsinstrument, als den Disponierten und als den Disponierenden zu erkennen. Die Burnout-Betroffenen sollen sich als Betroffene anerkennen und gleichzeitig als Experte in eigener Sache anrufen und entsprechend behandeln (lassen). Damit wird auch der mögliche und erwünschte Widerstand in die eigene Regie übernommen und auf sich selbst gerichtet. Nicht mehr die gesellschaftlichen Strukturen und immanenten Regierungsweisen sollen offenbart und bekämpft werden, sondern der Kampf soll mit den eigenen Lebensmustern, Denkstrukturen, Emotionen und Körpern geführt werden. Hier ließe sich weiter fragen, inwiefern der Neoliberalismus die Kritik und Widerstände tatsächlich in sein »Raster integriert « (Mönch 2018: 314) und damit »jegliche Kontingenz der Subjekte« (a.a.O.: 315) produktiv wendet »und selbst zur Ware bzw. zum Produktionsfaktor« macht (ebd.). Wirkt er nicht eher dadurch effektiver und leiser, 
dass er auf der einen Seite prekäre Lebens- und Regierungsverhältnisse installiert, die die Subjekte nicht imstande sind aufrechtzuerhalten, und auf der anderen Seite seine Unterstützung in Form einer ausbalancierten, den eigenen Ansprüchen geeigneten und nachhaltig produktiven Lebensweise anbietet? Schafft damit der Neoliberalismus gleichzeitig Prekarität und Lösungswege? Und ergänzt gerade die Prävention als immanente Regierungsweise die neoliberalen Ansprüche an grenzenlose Leistungssteigerung, die die Subjekte zur selbstgewollten Selbstausbeutung treibt, indem sie die möglichen Schäden vorauszusehen und zu kompensieren sucht?

Die Machteffekte der Burnout-Prävention werden schließlich auch dort sichtbar, wo Widerstand geleistet wird. Wie dies in dem methodologischen Teil angedeutet wurde, lassen sich drei Weisen des Widerstands analytisch voneinander trennen.

\subsection{Ein vordefinierter Widerstand}

Angesichts des Widerstands, der von den immanenten Regierungsweisen vordefiniert wird und daher den Subjekten als natürlich erscheint, lässt sich Folgendes feststellen. Zum einen erscheint durch die Verlagerung der Verantwortung in die Regie der Subjekte der Widerstand als eine individuelle Entscheidung: »Arbeitnehmer_innen wechseln den Arbeitsplatz, reduzieren ihre Arbeitszeit und damit auch ihre Ansprüche auf Status, Bezahlung oder Karrierechancen." (Menz et al. 2011 zit.n. Brunnett 2018: 339) Zum anderen stehen den Betroffenen und Gefährdeten zahlreiche Präventionsangebote zur Verfügung. Demnach neutralisiert die natürliche Sorge um sich - wer würde denn die eigene Gesundheit riskieren? den Widerstand gegen präventive Maßnahmen. Sich gegen die Prävention zu stellen würde heißen, sich selbst bewusst zu gefährden und damit den eigenen gesellschaftlichen Ausschluss selbst zu vollziehen. In dieser Selbst-Gefährdungs-SelbstErlösungs-Falle manifestiert sich das Wirken des Präventionsdispositivs, das, wie bei allen anderen Dispositiven, stets verschleiert und der hautnahen Erfahrung entfernt bleibt: »Regieren ist keine >lineare〈 Angelegenheit. Dispositive wirken immer nur auf Distanz, auch wenn Distanzen variieren. (Fach 2008: 107 [Herv. i. O.]) Das Regieren auf Distanz gelingt dem Präventionsdispositiv dadurch, dass (auch) in ihm »eine Umschreibung gesellschaftlicher Herrschaftsverhältnisse in individuelle Probleme stattfindet - insofern Konfliktverhältnisse und gesellschaftliche Widersprüche als individuelle, psychische Unzulänglichkeiten deklariert, diskutiert und von den als defizitär markierten Individuen in der Regel auch so empfunden werden. Wenn man so will, hat man es somit in summa mit einer Politik der Entpolitisierung zu tun« (Rau 2018: 316), weshalb die Begründung der Prävention »als Verhinderungshandeln immer prekär bleiben [muss].« (Kessl 2005: 144) 


\subsection{Ein schützender Widerstand}

Im Hinblick auf den Widerstand, den das Präventionsdispositiv gegen seine eigene Auflösung leistet, lassen sich folgende Beobachtungen festhalten. Einerseits handelt es sich beim Burnout um »eine >Grundproblematik^, mit der (wir) Menschen $\mathrm{zu}$ allen Zeiten und überall auf der Welt zu kämpfen hatten und haben." (Wenninger 2015: 16 [Herv. i. O.]) Die Erschöpfung und das Gefühl der Kraftlosigkeit gehören somit zu den fast alltäglichen Erfahrungen, die im Leben vorkommen, was ihre Behandlung nicht problematisch erscheinen lässt. Andererseits werden eben durch diese Alltäglichkeit die Belastung und der Stress auch aufgrund ihrer positiven Konnotation geschätzt:

»Über viel Stress und hohe Belastungen zu klagen, im Beruf und überhaupt im Leben, gehört in unserer leistungsfixierten Welt inzwischen zum guten Ton: 100 Mails habe ich pro Tag zu beantworten $-80.000 \mathrm{~km}$ pro Jahr bin ich auf der Straße-ich leiste jeden Monat 20 Überstunden! In jeder Klage schwingt aber auch >Stolz « - Erschöpfungsstolz - mit. An seine Leistungsgrenzen zu gehen adelt: Ich bin gefragt, weil jederzeit erreichbar. Es wertet mich auf, weil ich unabkömmlich, wichtig, der Schnellste, die Beste bin.« (a.a.O.: 19 [Herv. i. O.])

Somit schützt sich das Präventionsdispositiv vor seiner Hinterfragung von allen Seiten - Vorbeugen reicht nie und kommt nie rechtzeitig genug und gleichzeitig darf es keine Irritationen hervorrufen, den Arbeitsaufwand und die eigene Arbeitsstelle bedrohen und erst recht nicht das Profil eines erfolgreichen neoliberalen Subjekts in Frage stellen. Die Prävention zu bedrohen würde bedeuten, die Sicherungsmechanismen der gesellschaftlichen Lebenslage auszuschalten, diese einem unbekannten Ausmaß an Verletzlichkeit auszusetzen und damit die existenziellen Bedürfnisse aufs Neue austarieren zu müssen. Und gerade »[d]ie alltäglichen Ängste und Beunruhigungen der Menschen bilden das Scharnier zwischen existenziellen Bedürfnissen und gouvernementalen Interventionen." (Eisch-Angus 2019: 60) Das Präventionsdispositiv operiert an den Widersprüchen, die es selber herstellt. Das Unbekannte wird hergestellt, damit es präventiert werden kann. Die Unsicherheit wird erzeugt, damit sie gegen Sicherheit ausgetauscht werden kann. Die existenziellen Bedürfnisse werden bedroht, damit die Subjekte durch Vorbeugung vor dem Untergang geschützt werden. Und gleichzeitig breitet sich »die Dunkelsphäre von Angst und Verunsicherung [...] umso intensiver aus[...], je sichtbarer für Sicherheit gesorgt wird.«(a.a.O.: 573) Diese prozessierende Zementierung der Prävention nährt sich an den selbst produzierten Problemen, die die Subjekte als ihre eigenen erkennen sollen. 


\subsection{Ein möglicher Widerstand}

Schließlich, bezogen auf den Widerstand, den die disponierten Subjekte im Dispositiv leisten, d.h. sich dem Präventionsdispositiv widersetzen und ihm ungehorsam werden (können), lassen sich, bezogen auf Michel Foucault, vor allem zwei Formen benennen. Die erste Form antizipiert ein kollektives Gegen-Verhalten, das in Zeiten des Neoliberalismus auf dem Aufbau alternativer Regierungsordnungen beruht: "Die Etablierung alternativer Institutionen in den Bereichen der Bildung, des Gesundheitswesens und einer solidarischen Ökonomie heben sich deutlich von der neoliberalen Staatsdoktrin ab.« (Kastner 2008: 45) Durch die bisher immer noch äußerst schwammige Vorstellung von dem, wie die Burnout-Prävention tatsächlich wirkt und welche Machteffekte mit ihr entstehen, beschränkt sich diese Widerstandsform eher auf Selbsthilfegruppen oder wird von den Arbeitnehmerverbänden als Coaching oder Beratung auf individueller Ebene betrieben:

»Als Arbeitnehmerverband nehmen wir unsere Verantwortung wahr und engagieren uns für das Wohl der Angestellten. Weil das Thema Burnout und Stress so akut und verbreitet ist, bieten wir mit unseren Kooperationspartnerinnen von rundum achtsam eine eigene Themenreihe mit 3 Veranstaltungen an. Einmalig dabei ist deren ganzheitlicher Betrachtungsansatz - mit Körper, Geist und Psyche. Mit breitem fachlichem Know-how und eigenen Erfahrungen unterstützen sie vertrauensvoll und diskret.« (Rundum achtsam 2019)

Die zweite Form des Widerstands plädiert für zivilen Ungehorsam, eine Desertion, die »auf das Nicht-Befolgen von Befehlen, das Brechen von Normen oder ganz einfach die Verweigerung von Zustimmung« abzielt (Kastner 2008: 46). Die möglichen Umdeutungs- und Widerstandspotentiale dieser Art wurden bereits in vorherigen Teilen formuliert. Allerdings verbirgt sich in der veränderten Selbstführung bzw. Subjektivierungsweise das Risiko, »vom Neoliberalismus aufgenommen und funktionalisiert zu werden « (Bröckling 2007: 40f zit.n. Mönch 2018: 330), was den Widerstand umkehrt und die Kräfte noch mehr erschöpft. Das besagt im Weiteren, dass diese Art des Widerstands immer mit einer Macht, sich gegen den anderen - auch gegen ein immanentes Regierungsregime wie die Burnout-Prävention wenden zu können, operieren muss. Das Ziel der Macht, wie sie im Rückgriff auf Friedrich Nietzsche Michel Foucault gedacht und analytisch gebraucht hat, besteht jedoch darin, »den Widerstand der entgegenstehenden Macht nicht nur zu erfahren, sondern zu brechen (>überwinden`), um die eigene Stärke zu steigern. (Klass 2008: 153 [Herv. i. O.]) Nun erscheint auch diese Form des Widerstands bei den Burnout-Betroffenen bzw. -Gefährdeten als äußerst problematisch, denn sie verfügen nicht über Macht, weder als physische Kraft noch als psychisches Vermögen, mit der sie sich wehren, widersetzen oder Ungehorsam leisten können. Es stellt sich daher die Frage, ob Widerstand an dem Nullpunkt, in der Erfahrung der leib- 
lichen und psychischen Ohnmacht, beginnen und wurzeln kann. Um dieser Frage nachzugehen, wird eine kurze Passage aus einer Burnout-Ratgeberliteratur zitiert, die das Thema Ohnmacht behandelt:

»Manchmal kämpfen wir gegen Windmühlen. Manchmal waren all die Mühen, all der Aufwand, all die Arbeit umsonst. All das hat nichts genutzt und nichts gebracht. Wir sind bis an unsere Grenzen gegangen und haben doch unser Ziel nicht erreicht. Ein Gefühl der Ohnmacht kann sich schnell in uns breitmachen. Wir fühlen uns schwach und leer. Wer kennt dieses Gefühl nicht? Es ist sehr unangenehm und belastend. Und doch kann gerade dieses Cefühl uns Demut lehren. Es kann mit dazu beitragen, dass wir als Menschen, als Persönlichkeiten wachsen und reifen und zu mitfühlenden, verständnisvollen und warmherzigen Menschen werden. Wer das Gefühl der Ohnmacht und Hilflosigkeit kennt, der hebt nicht ab, der bleibt sauf dem Teppich`, der läuft nicht Gefahr, überheblich und arrogant zu werden. Ohnmacht ist keine schöne, aber doch eine sinnvolle und wichtige Erfahrung.«(Gesing 2017: 60f [Herv. i. O.])

Die Ohnmacht tritt hier als belastend und doch als lehrreich auf. Als Konsequenz einer übermäßigen Anstrengung gegenüber Menschen und Tatsachen, die nicht zu bewältigen sind, und gleichzeitig als Möglichkeit, das Lebensschicksal wenden und neue Wege bahnen zu können. Als eine unangenehme Erfahrung, die Schwäche, Kontrollverlust und Leere spüren lässt, die aber Einhalt gebietet und den Weg zum Neubeginn weist. Das Gefühl der Ohnmacht begleitet die Erfahrung eines jeden Betroffenen. Und gleichzeitig bringt es das Negativbild des Neoliberalismus zum Erscheinen: »antriebslos, emotional erstarrt statt flexibel, zurückgezogen statt vernetzt, ohnmächtig statt ermächtigt, selbstzweifelnd statt selbstbewusst - konstitutionell überfordert.« (Bröckling 2007: 289ff zit.n. Mönch 2018: 335) Wenn das Präventionsdispositiv als immanente Regierungsweise versucht, die gesellschaftlichen Problemlagen auf die Subjekte zu übertragen, können umgekehrt auch die Lebenssituationen der Burnout-Betroffenen etwas über das Präventionsdispositiv und das dahinterstehende neoliberale politische Projekt sagen? Wessen Ohnmächtigskeitsgefühle werden durch die Subjekte erlebt und inszeniert? Signalisiert die Müdigkeit des Subjekts nicht eher die Ohnmacht des Regimes? Vor wessen Erschöpfung soll dann die Prävention schützen?

Ohnmächtig zu sein und einen Widerstand zu leisten scheint daher widersprüchlich zu sein. Doch, die Erschöpfung und Müdigkeit sind zugleich Erfahrungen, d.h. Momente, in denen Altes hinter sich gelassen wird und Neues entstehen kann, wo das Bestehende auf den Prüfstand gestellt wird. Die Müdigkeit inspiriert: »Die Inspiration der Müdigkeit sagt weniger, was zu tun ist, als was gelassen werden kann.«(Handke 1989: 74 zit.n. Böhme 2018: 28) Gerade diese inspirierende ")Müdigkeit der negativen Potenz«, nämlich des >nicht-zu« (Han 2010: 60 [Herv. i. O.]), widersteht dem erlernten Drang, »sofort [zu] reagieren und jedem Impuls 
[zu] folgen« (a.a.O.: 39f), der »bereits eine Krankheit, ein Niedergang, ein Symptom der Erschöpfung « ist (ebd.). Diese inspirierende Müdigkeit »als Kunst des Gelassen-Seins, das heißt, dem Sein näher als dem Gestell und dem Gerede des Man, wie Heidegger sagen würde« (Böhme 2018: 28), eröffnet neue Denkräume, erschließt Möglichkeiten, die sich im Leistungs- und Wachstumsmodus erschöpft haben. Mit Gilles Deleuze gesprochen, kündigt die Erfahrung der Ohnmacht als inspirierende Müdigkeit eine Politik des Schweigens an:

»Die Schwierigkeit ist heute nicht mehr, dass wir unsere Meinung nicht frei äußern können, sondern Freiräume der Einsamkeit und des Schweigens zu schaffen, in denen wir etwas zu sagen finden. Repressive Kräfte hindern uns nicht mehr an der Meinungsäußerung. Im Cegenteil, sie zwingen uns sogar dazu. Welche Befreiung ist es, einmal nichts sagen zu müssen und schweigen zu können, denn nur dann haben wir die Möglichkeit, etwas zunehmend Seltenes zu schaffen: Etwas, das es tatsächlich wert ist, gesagt zu werden.« (Deleuze 1995: 129 zit.n. Hardt \& Negri 2013: 21)

Erschöpfung macht schweigend. Nicht einmal den Schrei des Ärgers und der Wut lässt der kraftlose Zustand der Burnout-Betroffenen zu. Und doch, das ohnmächtige Schweigen und die provozierende Stille machen das Gegenüber, das immanente Machtregime, hilflos. Was ihm bleibt, ist die Zuflucht »bei reinem Zwang oder schlichter Gewalt« (Foucault [1982] 2005: 287) zu suchen. Ohnmacht als Ausdruck der Freiheit zu verstehen, die sich der Machtausübung widersetzen will (vgl. ebd.), heißt dann, die Macht über sich selbst als Zielscheibe und Produktionsfaktor des immanenten (neoliberalen) Regierens nicht abgeben zu wollen.

\section{Regieren durch Vorbeugen}

Als drittes haben sich die kritischen Auseinandersetzungen mit der BurnoutPrävention darum bemüht, die Mechanismen des Regierens durch Vorbeugen zu entschlüsseln und dessen charakteristische Wesenszüge zu hinterfragen. Diese lassen sich auf der Mikro- und Makroebene beobachten sowie anhand von drei Aspekten - die Zeit, der Körper und das Nicht-Existente - unterscheiden.

\subsection{Regieren auf der Mikroebene}

Wie die Analysen nahegelegt haben, wirkt das immanente Regierungsregime der Prävention vor allem auf der individuellen Mikroebene. Die Subjekte der Prävention werden produziert, angesprochen, angeleitet und präventiert bzw. zur SelbstPrävention geführt. Hier wird die Verschränkung mit anderen Dispositiven bzw. ihren Teilen besonders anschaulich. Beispielhaft zeigt das Niklas Rose in seiner 